\title{
Proteomics as a tool for the modelling of biological processes and biomarker development in nutrition research
}

\author{
Baukje de Roos* and Harry J. McArdle \\ Division of Vascular Health, Rowett Research Institute, Greenburn Road, Bucksburn, Aberdeen AB21 9SB, United Kingdom
}

Nutrition research has slowly started to adopt the proteomics techniques to measure changes in the protein complement of a biological system. This enables modelling of biological processes in response to dietary interventions, as well as the elucidation of novel biomarkers for health or disease that are sensitive to such interventions. There are limited studies on the effect of micronutrients on the proteome, so this review concentrates rather more on dietary intervention studies that have used proteomics (mainly classical 2D gel electrophoresis combined with mass spectrometry) to elucidate changes in pathways that relate to glucose and fatty acid metabolism, oxidative stress, anti-oxidant defence mechanisms and redox status. The ability to measure regulation of more low abundant proteins, such as those involved in inflammatory pathways, as well as the evaluation and validation of newly discovered candidate biomarkers in human biofluids, may depend on the introduction of more quantitative and sensitive methods like multiple reaction monitoring (MRM) and multiplexed immunoassays in nutrition research.

Proteomics: Biomarker: Nutrition: Metabolism: Oxidative stress: Inflammation

Epidemiological studies show strong associations between diet and the incidence and severity of disease ${ }^{(1-4)}$. However, the identification of the bioactive components from diets and foodstuffs and defining their mode of action is challenging. Diets are complex, consisting of many separate food items, and each food item itself is a complex mix of bioactive components ${ }^{(5)}$. Their interactions with physiological systems, therefore, are very difficult to deduce using traditional experimental methods. The global nature of nutrigenomics technology is a real advantage for elucidating the complex physiological effects of food components, nutrients, or a specific diet, which are often mediated through multiple biochemical and molecular mechanisms. Furthermore, knowledge about the actions of foods and nutrients on all possible physiological outcomes related to, for example, chronic disease development, is essential to identify dietary strategies that will promote health and delay the onset of chronic diseases.

The potential value of proteomics for nutritional sciences has been recognised for some years and many authors have written excellent reviews (see, for example ${ }^{(6-10)}$ ) promulgating its use in nutrition. However, the total number of reviews published up to 2007 actually exceeds the number of research papers on proteomics! (Fig. 1). When searching Pubmed, using the search terms (proteomic*) and (nutrition* or diet* or food), and eliminating all proteomics-related publications that i) were not published in English; ii) were not dealing with an in vivo or in vitro dietary intervention, or iii) were based on plant/food/crop proteomics only, we found 88 reviews and only 73 research papers. Thus, whilst proteomics is presented as an emerging and promising tool that enables the elucidation of mechanisms of action as well as development of relevant biomarkers of health or disease, the actual use of this technique in dietary intervention trials is still rather limited. In relation to micronutrient metabolism, there are only a few papers examining either the proteomic consequences of dietary treatment or the potential for proteomics to be used as a biomarker for status. In this review, therefore, we have also included other dietary treatments, that can be used as examples of the method and hopefully provide inspiration to others to adopt the techniques in their micronutrient research.

\section{Proteomics as a tool to elucidate mechanisms of action}

Proteomics has the advantage over cDNA micro-arrays in that it measures the functional product (protein) of gene expression, and allows the identification of modifications that may relate to the activation or inactivation of proteins by dietary

Abbreviations: 2D gel electrophoresis, two-dimensional gel electrophoresis; (Apoe ${ }^{-1}$ ) mice, apolipoprotein E knockout mice; cyGPx, cytosolic glutathione peroxidase; CHD, coronary heart disease; CLA, conjugated linoleic acid; Cu/Zn SOD, copper/zinc superoxide dismutase; CV, coefficient of variation; DIGE, difference gel electrophoresis; GAPDH, glyceraldehyde-3-phosphate dehydrogenase; GST, glutathione-S-transferase; HDL, high density lipoprotein; HNE, 4-hydroxynonenal; HSP 70kD, heat shock protein $70 \mathrm{kD}$; ICAT, isotope-coded affinity tags; iTRAC, isobaric tag relative absolute protein quantitation; LC-MS/MS, liquid chromatography/mass spectrometry/mass spectrometry; MALDI-TOF, matrix assisted laser desorption/ionization-time of flight; MRM, multiple reaction monitoring; MS, mass spectrometry; NAD, nicotinamide adenine dinucleotide; NF-кB, nuclear factor-kappa B; 3-NT, 3-nitrotyrosine; NuGO, European Nutrigenomics Organisation; PPAR $\alpha$, peroxisome proliferator activated receptor alpha; PBMC, peripheral blood mononuclear cell; SELDI, surface enhanced laser desorption/ionisation time of flight mass spectroscopy; SREBP, sterol regulatory element binding protein; TBARS, thiobarbiturate reactive substances.

* Corresponding author: Dr Baukje de Roos, fax +44 (0)1224 716629, email b.deroos@rowett.ac.uk 


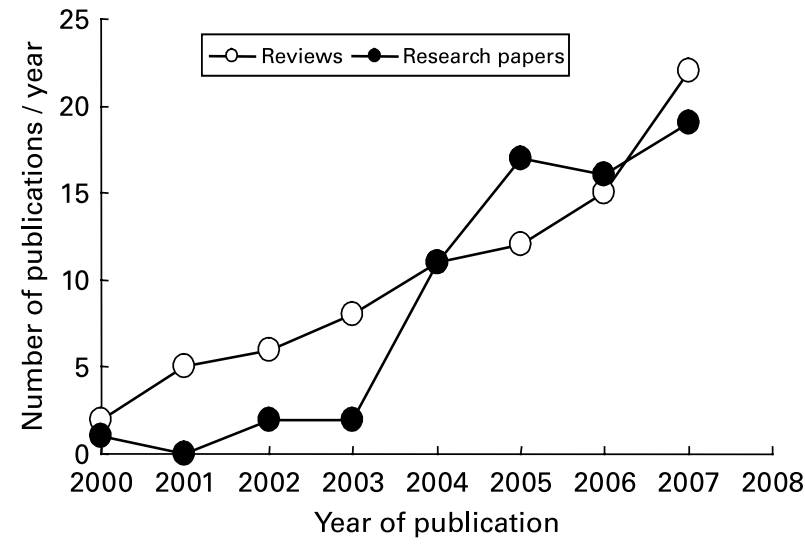

Fig. 1. Reviews and research papers relating to proteomics, nutrition, diet and food published from 2000-2007.

interventions. Such proteins may not only play a major physiological role in target organs, but could also reflect changes in mechanisms initiated by dietary intervention when circulating in the blood.

Classical two-dimensional (2D) gel electrophoresis, coupled with spot analysis by mass spectrometry, is still the most widely used technical approach in proteomics to identify changes in individual proteins of tissues, cells and biofluids upon nutritional intervention ${ }^{(7,6)}$. While this method is one of the most labour-intensive of the several types of $2 \mathrm{D}$ separation methods available, it actually yields a physical separation of intact polypeptides from each other, providing information about molecular weight and iso-electric point, parameters that can be used to narrow down the identification of the protein ${ }^{(11)}$. However, the dynamic range of protein concentrations spans six orders of magnitude in a cell, and even 10 in the human body (for example in plasma). Proteins involved in inflammatory pathways, like cytokines, are secreted and circulate in the body at a concentration nine orders of magnitude less than albumin ${ }^{(12)}$. It is currently difficult to visualise and detect differential regulation of low abundant, very hydrophobic, acidic or basic proteins ${ }^{(13)}$. Therefore, 2D gel electrophoresis may not always represent the most sensitive tool to reveal effects of nutritional interventions on inflammatory pathways. On the other hand, 2D gel electrophoresis and mass spectrometry has established itself as a valuable tool in elucidating changes in pathways that relate to glucose and fatty acid metabolism as well as pathways relating to oxidative stress, anti-oxidant defence mechanisms and redox status $^{(14-16)}$.

Studies in our laboratory have demonstrated that two structurally almost identical dietary conjugated linoleic acid (CLA) isomers had divergent mechanistic effects on atherosclerosis development and insulin resistance in Apoe ${ }^{-/-}$ mice. Consumption of trans 10 , cis 12 CLA, a fatty acid present in supplements that are currently freely available, was pro-atherogenic and induced pathways involved in the development of insulin resistance, as evidenced by simultaneous changes in various enzymes involved in gluconeogenesis, glycolysis and ketogenesis ${ }^{(17)}$. Consumption of $\operatorname{cis} 9$, trans 11 isomer of CLA, on the other hand, was anti-atherogenic and specifically induced heat shock protein (HSP) $70 \mathrm{kD}$ protein levels ${ }^{(17)}$. Although one may consider this to be an indication of increased oxidative stress, we have postulated that up-regulation of HSP $70 \mathrm{kD}$ protein upon exposure to a relatively mild stress stimulus (like cis9, trans 11 CLA) could protect against exposure to more severe stress later, possibly including antiinflammatory pathways (mediated through $\mathrm{NF}-\kappa \mathrm{B})^{(18)}$. Indeed, increased levels of human serum HSP 70 have been associated with a low coronary artery disease risk, independent of traditional risk factors ${ }^{(19)}$.

Using proteomics, we were also able to show that the consumption of dietary fish oil and trans 10 , cis 12 CLA induced differential expression of long chain acyl-CoA thioester hydrolase protein as an indicator of $\beta$-oxidation of fatty acids in the liver, and the consumption of dietary fish oil, olive oil and trans 10 , cis 12 CLA induced differential expression of adipophilin protein as an indicator of selective hepatic lipid accumulation and triglyceride secretion ${ }^{(14,17)}$.

In a recent study we applied a systems biology approach to gain a better understanding of the mechanisms by which the fatty acids of olive oil, or its minor antioxidant constituents, may affect hepatic metabolic pathways, oxidative stress and eventually atherogenesis. Extra virgin olive oil reduced atherosclerotic plaque size after 10 weeks of intervention in apolipoprotein E knockout (Apoe ${ }^{-1-}$ ) mice but also induced the accumulation of triglycerides in the liver, resulting in an increased hepatic fat content. These results imply that olive oils can, on the one hand, reduce the risk of atherosclerotic plaque formation, but can also increase the risk of hepatic steatosis. A systems biology approach appeared to be crucial for unravelling the complex interactions. We found a significant up-regulation of a large array of antioxidant enzymes upon consumption of extra virgin olive oils that may diminish oxidative stress instigated by hepatic steatosis and in addition, may slow down the development of atherosclerosis. Therefore, compounds in extra virgin olive oils (such as the polyphenols) may well be able to delay the onset of atherosclerotic lesions by combatting oxidative stress. Indeed, the accumulation of triglycerides may not pose a major challenge to the liver, and represent a relatively safe way to store triglycerides, as long as the antioxidant capacity is adequate to prevent lipotoxicity ${ }^{(15)}$.

A combination of transcriptomics and proteomics was used to determine mechanisms of (supra-physiological concentrations of) dietary quercetin in the healthy distal colon of inbred F344 rats, and revealed that quercetin changed the cellular energy metabolism from glycolysis in the cytoplasm towards fatty acid degradation in the mitochondria. Cancer cells generally exhibit increased glycolysis and mitochondrial dysfunction, and in human colon tumours an increase in glycolysis combined with decreased fatty acid degradation as an energy source has been observed previously. Therefore, the quercetin-mediated glycolysis-to-fatty acid degradation shift in energy pathways could represent an additional underlying mechanism involved in quercetin's potency to inhibit induced colorectal carcinogenesis ${ }^{(20)}$.

A combination of transcriptomics and proteomics was also applied to explain the mechanisms underlying changes in hepatic lipid metabolism during zinc deficiency ${ }^{(21)}$. Zinc is involved in many aspects of normal physiology, including intermediary metabolism, hormone secretion pathways, and immune defence. Deficiency has severe consequences, but the mechanisms involved are largely unknown. The results of 
this study provided evidence for major changes in the expression of gene groups functionally linked to hepatic lipogenesis and lipolysis, with an inverse regulation that fostered the accumulation of triglycerides in liver, changing the hepatic fatty acid pattern, and reducing fatty acid oxidation. The experimental findings provided support for an unbalanced gene transcription control via peroxisome proliferator activated receptor alpha $(\mathrm{PPAR} \alpha)$, thyroid hormone, and sterol regulatory element binding protein (SREBP)-dependent pathways, which may explain most of the apparently pleiotropic effects of zinc deficiency on hepatic fat metabolism ${ }^{(21)}$.

Proteomics was used as a tool to explore biochemical alterations in the adult brain after developmental vitamin D deficiency. For this, vitamin D-deficient female rats were mated with vitamin D normal males. Pregnant females were kept vitamin D-deficient until birth whereupon they were returned to a control diet. At week 10, protein expression in the progeny's prefrontal cortex and hippocampus was compared with control. Developmental vitamin D deficiency led to long-lasting alterations in expression of brain proteins involved in mitochondrial, cytoskeletal and neuronal function. Specifically, 36 proteins were identified which were involved in, for example, oxidative phosphorylation, redox balance, cytoskeleton maintenance, calcium homeostasis, chaperoning and neurotransmission. An in silico survey of vitamin $\mathrm{D}$ target genes identified a putative vitamin $\mathrm{D}$ responsive element in the promoter region of 6 of these 36 proteins. Interestingly, many of the proteins found to be dysregulated in this study have also been identified in post-mortem and genetic studies in patients with schizophrenia or multiple sclerosis, the most prominent similarity being the dysregulation of mitochondrial proteins ${ }^{(22)}$.

A rat model of oxidative stress was used to investigate the mechanism(s) whereby salicylic acid modulates potentially pro-cancerous activity in the colon using a proteomic approach. Supplementation of salicylic acid $(1 \mathrm{mg} / \mathrm{kg}$ diet) resulted in a significant change in expression of 55 cytosolic proteins extracted from the distal colon milled in liquid nitrogen. The functions of these proteins related to protein folding, protein transport, redox balance, energy metabolism and cytoskeletal regulation. Partial least squares analysis was performed to identify associations between significantly altered proteins and biochemical indices of oxidative stress (plasma and colon thiobarbiturate reactive substances (TBARS)), prostaglandin levels and cytosolic glutathione peroxidase (cyGPx) activity data. Seven of the proteins were involved in the two major redox pathways of thioredoxin and glutathione, which implies that salicylic acid can regulate redox signalling via similar pathways to vitamin $\mathrm{E}$, but may do this indirectly through modulation of interactive components of the redox signalling system ${ }^{(23)}$.

A last example of a proteomics approach that illustrates the technique's ability to measure oxidative stress and metabolic changes is given in a paper published by Opii and colleagues $^{(24)}$. These authors investigated the effect of a long-term treatment with an antioxidant-fortified diet for $2 \cdot 8$ years (containing vitamin E, L-carnitine, DL-alpha-lipoic acid, vitamin $\mathrm{C}$, and spinach flakes, tomato pomace, grape pomace, carrot granules and citrus pulp) and/or a program of behavioural enrichment on oxidative damage in the parietal cortex. They used aged Beagles as a model for human aging as these dogs naturally develop cognitive deficits and accumulate brain pathology that is similar to aging humans. The combined treatment caused the greatest decrease in the oxidative stress biomarkers protein carbonyls, 3-nitrotyrosine (3-NT), and the lipid peroxidation product 4-hydroxynonenal (HNE). Using comparative proteomics they found that protein carbonyl levels of glutamate dehydrogenase [NAD (P)], glyceraldehyde-3-phosphate dehydrogenase (GAPDH), $\alpha$-enolase, neurofilament triplet $\mathrm{L}$ protein, glutathioneS-transferase (GST) and fascin actin bundling protein were significantly decreased and that the protein levels of $\mathrm{Cu} / \mathrm{Zn}$ superoxide dismutase ( $\mathrm{Cu} / \mathrm{Zn} \mathrm{SOD})$, fructose-bisphosphate aldolase $\mathrm{C}$, creatine kinase, glutamate dehydrogenase and glyceraldehyde-3-phosphate dehydrogenase were increased in brain of the combined treatment group. The increased expression of a number of antioxidant proteins, in particular $\mathrm{Cu} / \mathrm{Zn}$ SOD, correlated with improved cognitive function. These findings suggest that the anti-oxidant plus behavioural enrichment treatment decreases the levels of oxidative damage and improves the antioxidant reserve systems in the aging canine brain, and may contribute to improvements in learning and memory ${ }^{(24)}$.

\section{Proteomics as a tool for biomarker development}

Dietary intervention causes changes in proteins that are secreted or otherwise released into the blood. An enormous asset of the proteomics platform is, therefore, the quantitative, noninvasive analysis of human body fluids to determine the consequences of such interventions. Plasma or serum, and circulating cells such as lymphocytes and platelets, can be relatively easily obtained from blood ${ }^{(7,16)}$ and proteomics technologies have the potential to deliver biomarkers for health, to reveal early indications of disease risk, assist in differentiating dietary responders from non-responders, and to discover beneficial food components ${ }^{(9)}$. Currently the major problem with this approach seems to be reproducibility ${ }^{(25)}$. It is well known that highdimensional biological technologies, such as proteomics, are liable to errors from both biological and non- biological variability. Some causes may include reagent and gel variability, technical variations, temperature and dye/stain incorporation. Biological variability may arise from factors such as circadian rhythm, sex, age, and smoking status, many of which may be unmeasured and, indeed, unquantifiable ${ }^{(6,16)}$. These sources of variation may easily obscure the biological changes under investigation, and this has led some to argue that proteomic profiling of blood is a relatively immature technology in need of further refinement ${ }^{(26)}$.

The extent of the inter-individual variability of the human proteome remains unclear but is an important factor to try and quantify ${ }^{(27)}$. We are currently addressing this issue in a 'proofof-principle' initiative funded by the European Nutrigenomics Organisation (NuGO) Network of Excellence. This project aims to determine biological variability in plasma, saliva, urine, platelet and peripheral blood mononuclear cell (PBMC) proteomics in healthy individuals during baseline and after a fasting challenge. In addition, we recently systematically assessed analytical variation in 2D gel electrophoresis of a depleted plasma, platelet and peripheral blood mononuclear cell protein homogenate within and between four laboratories. 
We found that the within-laboratory coefficient of variation (CV) between gels (based on all visual spots on the 2D gel) ranged from $18-68 \%$ in four different laboratories, whereas the between-laboratory CV (based on 30 pre-selected abundant protein spots) ranged between $22-34 \%$. This variation must be considered when designing human intervention studies that use proteomics tools to elucidate the (often subtle) effects of dietary compounds on potential novel biomarkers of health. Several steps in the proteomics procedure appeared particularly critical for and contributed to this variation. Amongst these were reproducible depletion of plasma of its most abundant proteins, contamination of circulating cells with other cells from blood, use of tricine to improve the running conditions of the 2D gel electrophoresis procedure, acquisition of high resolution images for comparative analysis, and the careful assessment of spot identifications obtained by matrix assisted laser desorption/ionization-time of flight (MALDI-TOF) or liquid chromatography/mass spectrometry/ mass spectrometry (LC-MS/MS) analysis ${ }^{(16)}$.

Despite these problems, proteomics has a real potential for the development of biomarkers. In one of our recent trials we assessed the effects of daily fish oil for six weeks on the serum proteome using 2-DE. We found that serum levels of apolipoprotein A1, apolipoprotein L1, zinc- $\alpha$-2-glycoprotein, haptoglobin precursor, $\alpha$-1-antitrypsin precursor, anti-thrombin III-like protein, serum amyloid P component, and haemopexin were all significantly down-regulated (all $P<0.05$ ) by fish oil compared with high oleic sunflower oil supplementation. In addition, the decrease in serum apolipoprotein A1 was associated with a significant shift towards the larger, more cholesterol-rich high density lipoprotein $2\left(\mathrm{HDL}_{2}\right)$ particles. The alterations in serum proteins and HDL size imply that fish oil activates anti-inflammatory and lipid modulating mechanisms believed to impede the early-onset of coronary heart disease (CHD). These proteins are potential diagnostic biomarkers to assess the mechanisms whereby fish oils protect against CHD in humans ${ }^{(28)}$.

A proteomics approach was also used to identify alterations in PBMC proteins of healthy males ingesting flaxseed for a week. PBMC's from the same study subjects were also exposed to physiological concentrations of enterolactone (a metabolite produced from dietary lignans by colonic microflora) ex vivo to assess whether similar effects on the proteome could be observed as those caused by dietary flaxseed. Intervention with flaxseed in healthy males resulted in enhanced plasma enterolactone levels in all volunteers, although both baseline levels and maximal plasma concentrations varied considerably between subjects. In addition, a fairly robust change in 16 PBMC proteins was observed upon consumption of flaxseed. Four out of these sixteen proteins were altered in a similar manner when blood mononuclear cells were exposed ex vivo to enterolactone: enhanced levels of peroxiredoxin, decreased levels of the long-chain fatty acid $\beta$-oxidation multi-enzyme complex proteins, and levels of glycoprotein $\mathrm{III} / \mathrm{II}^{(29)}$.

Proteomics of PBMC's was also used to identify biomarkers of response to a dietary supplementation with an isoflavone extract for 8 weeks in postmenopausal women. Twenty-nine proteins, including several involved in the anti-inflammatory response, showed significantly altered expression in the mononuclear blood cells following the soy-isoflavone intervention. Heat shock protein 70 , a lymphocyte-specific protein phosphatase and proteins that promote increased fibrinolysis, such as $\alpha$-enolase, were all found at increased intensities, whereas those that mediate adhesion, migration, and proliferation of vascular smooth muscle cells, such as galectin-1, showed decreased expression. As no overall anti-inflammatory activity of the soy intervention was observed when clinically relevant inflammation markers were measured in plasma, it was suggested that the PBMC proteome may represent a more sensitive approach to detect inhibition of inflammatory processes and that may also respond earlier than those plasma markers classically used ${ }^{(30)}$.

\section{Future developments}

It is unlikely that a single protein, by itself, clearly defines a specific treatment effect distinguishing it from all other diseases or conditions ${ }^{(31)}$. Having several markers may allow a more definite diagnosis, better disease stratification and additional value. Some caution must be exercised, however. It has been suggested that a large number of correlated biomarkers (although underscoring the importance of a specific biological pathway) is substantially less informative and predictive than a small number of uncorrelated biomarkers ${ }^{(27)}$. Furthermore, when considering the importance of specific biological pathways, it is important to note that the combination of proteomics data with physiological parameters, using the powerful statistical tool of correlation analysis, will provide clearer insights into the mechanisms by which dietary components regulate metabolic processes and ultimately change phenotypes ${ }^{(10,14,17)}$.

To date, transcriptomics has been more readily embraced by the nutrition research community. However, as new and improved proteomics techniques emerge this may change ${ }^{(5)}$. So far, most applied proteomic methods have been based on 2D gel electrophoresis, which is undirected and semiquantitative. While differences based on abundance may look obvious in a given 2D gel or MS spectrum, obtaining accurate measures over multiple samples analysed at different times is more difficult. Furthermore, high-abundance proteins will be identified many more times than low-abundance proteins. Other approaches are beginning to be developed which may help to solve these problems ${ }^{(32)}$.

A recent advance is the introduction of difference gel electrophoresis (DIGE) technology, allowing for direct quantitative measurements between differentially labelled samples using cyanine fluorescent dyes prior to gel electrophoresis. When absolute protein variation between two or three samples is the primary target, this method is more reproducible and accurate and not limited by distortion from gel-to-gel variation $^{(33)}$, but it is costly and automation is difficult ${ }^{(34)}$. Shotgun proteomics (digestion of the protein mixture and multidimensional chromatographic separation of peptides followed by on-line mass spectrometric peptide detection and sequencing ${ }^{(8)}$ ) can also be combined with stable isotope labelling to allow for the quantification of changes in protein expression levels of hundreds to thousands of proteins in a single experiment. The most commonly adopted approaches include isotope-coded affinity tags $\left(\right.$ ICAT) ${ }^{(35)}$ and isobaric tag relative absolute protein quantitation (iTRAC) ${ }^{(36)}$ methods. However, quantification is based on relative changes in the levels of labelled peptides that may 
be common to a family of proteins, with differential regulation/ abundance, and therefore quantification experiments can lead to ambiguous or conflicting results ${ }^{(37)}$.

Recently, triple quadrupole-derived technologies combined with a new generation linear ion trap have enabled quantitative analysis of (known) specific peptides (including those that are post-translationally modified) in complex biological mixtures such as human plasma and serum with very high sensitivity and selectivity in the multiple reaction monitoring (MRM) mode. The high selectivity results from monitoring one pair of precursor/fragment ions characteristic of a single peptide. In addition, two levels of mass selection in MRM experiments result in a substantial increase in sensitivity, because the first filter only transmits a small ion population, and thus minimises the overall chemical background. By adding calibrated, isotopically labelled reference peptides, precise quantitative information can be obtained ${ }^{(38)}$. This approach represents a significantly faster and probably less expensive tool to quantitatively measure low abundance proteins in blood (present at low nanogram/milliliter concentrations) that can be highly 'multiplexed,' permitting the simultaneous measurement of multiple proteins. Furthermore, the within-run coefficients of variation are between $2-22 \%$, which is significantly lower than other proteomic approaches ${ }^{(39)}$. Such an approach may provide better opportunities to observe subtle changes in for example inflammatory pathway-related protein markers upon dietary intervention. Alternatively, arrays of spotted antibodies have become available as a new tool for protein profiling. Many such arrays focus on plasma proteins such as cytokines and adipokines, which are protein classes of interest for nutrigenomics studies ${ }^{(40)}$. This technology will develop in the coming years, both in performance and in the number of proteins that can be analysed simultaneously ${ }^{(41)}$.

SELDI (surface enhanced laser desorption/ionisation time of flight mass spectroscopy) has had a mixed press in terms of value for high throughput proteomics. In theory, it should be an excellent system. It uses differentially chemically modified surfaces to bind proteins or peptides of interest. These surfaces include anion and cation exchangers, immobilised metal affinity surfaces, hydrophobic and antibody binding, among others. The proteins are eluted using lasers of different strengths and the peptides separated on a time of flight mass spectrometer. The spectra can be compared for differences between conditions. The system has been used extensively in pharmacological and health and disease comparisons ${ }^{(42)}$, but much less so in nutritional treatments ${ }^{(43,44)}$ (Miller, Gambling, Araya and McArdle, unpublished data). However, there are some problems, especially in translating the technology from the laboratory to the field or bedside. This is well reviewed by Reymond and Schlegel ${ }^{(45)}$. Many of these problems are shared by other proteomics technologies, since they relate to such things as storage of samples, variations between patients, subtle changes in disease state or progress, diurnal variation and age. However, the extra sensitivity of SELDI means that it is more liable to give false positives or negatives, so careful statistical analysis is essential.

\section{Conclusion}

Current proteomics approaches in nutrition research mainly include the use of protein separation, visualisation and identification by $2 \mathrm{D}$ gel electrophoresis combined with mass spectrometry. Despite the few number of studies that have been undertaken so far, and despite its limited ability to detect regulation of low abundant proteins, this approach has already provided valuable insights in the effects of several dietary interventions on (relatively abundant) proteins involved in the regulation of glucose and fatty acid metabolism, oxidative stress, and the redox system. The success of proteomics in nutrition research will depend on the introduction and adaptation of more quantitative and sensitive methods, which are currently already being validated and applied in the pharmaceutical industry. Such methods will enable the development of assays to evaluate and validate newly discovered candidate biomarkers in human biofluids.

\section{Acknowledgements}

The authors' work is funded by the Scottish Government Rural and Environment Research and Analysis Directorate (RERAD), the European Union (NuGO) and the International Copper Association.

\section{References}

1. Hu FB \& Willett WC (2002) Optimal diets for prevention of coronary heart disease. JAMA 288, 2569-2578.

2. Van Horn L, McCoin M, Kris-Etherton PM, Burke F, Carson JA, Champagne CM, Karmally W \& Sikand G (2008) The evidence for dietary prevention and treatment of cardiovascular disease. $J$ Am Diet Assoc 108, 287-331.

3. Riboli E \& Norat T (2003) Epidemiologic evidence of the protective effect of fruit and vegetables on cancer risk. Am J Clin Nutr 78, 559S-569S.

4. World cancer research Fund AIfCR (editors) (2007) Food, Nutrition, Physical Activity, and the Prevention of Cancer: a Global Perspective. Washington: World Cancer Research Fund

5. Brown L \& van der OF (2007) Nutritional genomics: food industry applications from farm to fork. Br J Nutr 97, 1027-1035.

6. Kim H, Page GP \& Barnes S (2004) Proteomics and mass spectrometry in nutrition research. Nutrition 20, 155-165.

7. Fuchs D, Winkelmann I, Johnson IT, Mariman E, Wenzel U \& Daniel H (2005) Proteomics in nutrition research: principles, technologies and applications. Br J Nutr 94, 302-314.

8. Kussmann M \& Affolter M (2006) Proteomic methods in nutrition. Curr Opin Clin Nutr Metab Care 9, 575-583.

9. Kussmann M, Raymond F \& Affolter M (2006) OMICS-driven biomarker discovery in nutrition and health. J Biotechnol 124, $758-787$.

10. Milner JA (2007) Nutrition in the 'omics' era. Forum Nutr $\mathbf{6 0}$, $1-24$.

11. Barnes S \& Kim H (2004) Nutriproteomics: identifying the molecular targets of nutritive and non-nutritive components of the diet. J Biochem Mol Biol 37, 59-74.

12. Kenyon GL, DeMarini DM, Fuchs E, Galas DJ, Kirsch JF, Leyh TS, Moos WH, Petsko GA, Ringe D, Rubin GM \& Sheahan LC (2002) Defining the mandate of proteomics in the post-genomics era: workshop report. Mol Cell Proteomics 1, 763-780.

13. Gygi SP, Corthals GL, Zhang Y, Rochon Y \& Aebersold R (2000) Evaluation of two-dimensional gel electrophoresisbased proteome analysis technology. Proc Natl Acad Sci U S A 97, 9390-9395.

14. de Roos B, Duivenvoorden I, Rucklidge G, Reid M, Ross K, Lamers RJ, Voshol PJ, Havekes LM \& Teusink B (2005) 
Response of apolipoprotein E*3-Leiden transgenic mice to dietary fatty acids: combining liver proteomics with physiological data. FASEB $J$ 19, 813-815.

15. Arbones-Mainar JM, Ross K, Rucklidge GJ, Reid M, Duncan G, Arthur JR, Horgan GW, Navarro MA, Carnicer R, Arnal C, Osada J \& Roos BD (2007) Extra Virgin Olive Oils Increase Hepatic Fat Accumulation and Hepatic Antioxidant Protein Levels in APOE(-/-) mice. J Proteome Res 6, 4041-4054.

16. de Roos B, Duthie SJ, Polley AC, Mulholland F, Bouwman F, Heim C, Rucklidge G, Johnson IT, Mariman E, Daniel H \& Elliott RM (2008) Proteomic methodological recommendations for studies involving human plasma, platelets and peripheral blood mononuclear cells. J Proteome Res, May 20 [Epub ahead of print].

17. de Roos B, Rucklidge G, Reid M, Ross K, Duncan G, Navarro MA, Arbones-Mainar JM, Guzman-Garcia MA, Osada J, Browne J, Loscher CE \& Roche HM (2005) Divergent mechanisms of cis 9, trans11-and trans 10 , cis12-conjugated linoleic acid affecting insulin resistance and inflammation in apolipoprotein $\mathrm{E}$ knockout mice: a proteomics approach. FASEB J 19, 1746-1748.

18. Shimizu M, Tamamori-Adachi M, Arai H, Tabuchi N, Tanaka H \& Sunamori M (2002) Lipopolysaccharide pretreatment attenuates myocardial infarct size: a possible mechanism involving heat shock protein 70 -inhibitory kappaBalpha complex and attenuation of nuclear factor kappaB. J Thorac Cardiovasc Surg 124, 933-941.

19. Zhu J, Quyyumi AA, Wu H, Csako G, Rott D, Zalles-Ganley A, Ogunmakinwa J, Halcox J \& Epstein SE (2003) Increased serum levels of heat shock protein 70 are associated with low risk of coronary artery disease. Arterioscler Thromb Vasc Biol 23, 1055-1059.

20. Dihal AA, van der WH, Hendriksen PJ, Charif H, Dekker LJ, Ijsselstijn L, de Boer VC, Alink GM, Burgers PC, Rietjens IM, Woutersen RA \& Stierum RH (2008) Transcriptome and proteome profiling of colon mucosa from quercetin fed F344 rats point to tumor preventive mechanisms, increased mitochondrial fatty acid degradation and decreased glycolysis. Proteomics 8, 45-61

21. Tom DH, Doring F, Fuchs D, Roth HP \& Daniel H (2005) Transcriptome and proteome analysis identifies the pathways that increase hepatic lipid accumulation in zinc-deficient rats. J Nutr 135, 199-205.

22. Almeras L, Eyles D, Benech P, Laffite D, Villard C, Patatian A, Boucraut J, Mackay-Sim A, McGrath J \& Feron F (2007) Developmental vitamin D deficiency alters brain protein expression in the adult rat: implications for neuropsychiatric disorders. Proteomics 7, 769-780.

23. Drew JE, Padidar S, Horgan G, Duthie GG, Russell WR, Reid M, Duncan G \& Rucklidge GJ (2006) Salicylate modulates oxidative stress in the rat colon: a proteomic approach. Biochem Pharmacol 72, 204-216.

24. Opii WO, Joshi G, Head E, Milgram NW, Muggenburg BA, Klein JB, Pierce WM, Cotman CW \& Butterfield DA (2008) Proteomic identification of brain proteins in the canine model of human aging following a long-term treatment with antioxidants and a program of behavioral enrichment: relevance to Alzheimer's disease. Neurobiol Aging 29, 51-70.

25. Astle J, Ferguson JT, German JB, Harrigan GG, Kelleher NL, Kodadek T, Parks BA, Roth MJ, Singletary KW, Wenger CD \& Mahady GB (2007) Characterization of proteomic and metabolomic responses to dietary factors and supplements. $J$ Nutr 137, 2787-2793.

26. Coombes KR, Morris JS, Hu J, Edmonson SR \& Baggerly KA (2005) Serum proteomics profiling-a young technology begins to mature. Nat Biotechnol 23, 291-292.
27. Gerszten RE \& Wang TJ (2008) The search for new cardiovascular biomarkers. Nature 451, 949-952.

28. de Roos B, Geelen A, Ross K, Rucklidge G, Reid M, Duncan G, Caslake M, Horgan G \& Brouwer IA (2008) Identification of potential serum biomarkers of inflammation and lipid modulation that are altered by fish oil supplementation in healthy volunteers. Proteomics 8, 1965-1974.

29. Fuchs D, Piller R, Linseisen J, Daniel H \& Wenzel U (2007) The human peripheral blood mononuclear cell proteome responds to a dietary flaxseed-intervention and proteins identified suggest a protective effect in atherosclerosis. Proteomics 7, 3278-3288.

30. Fuchs D, Vafeiadou K, Hall WL, Daniel H, Williams CM, Schroot JH \& Wenzel U (2007) Proteomic biomarkers of peripheral blood mononuclear cells obtained from postmenopausal women undergoing an intervention with soy isoflavones. Am J Clin Nutr 86, 1369-1375.

31. Vasan RS (2006) Biomarkers of cardiovascular disease: molecular basis and practical considerations. Circulation 113, 2335-2362.

32. Drake TA \& Ping P (2007) Thematic review series: systems biology approaches to metabolic and cardiovascular disorders. Proteomics approaches to the systems biology of cardiovascular diseases. J Lipid Res 48, 1-8.

33. Issaq HJ \& Veenstra TD (2007) The role of electrophoresis in disease biomarker discovery. Electrophoresis 28, 1980-1988.

34. Kussmann M (2007) How to comprehensively analyse proteins and how this influences nutritional research. Clin Chem Lab Med 45, 288-300.

35. Smolka MB, Zhou H, Purkayastha S \& Aebersold R (2001) Optimization of the isotope-coded affinity tag-labeling procedure for quantitative proteome analysis. Anal Biochem 297, $25-31$.

36. Streckfus CF, Mayorga-Wark O, Arreola D, Edwards C, Bigler L \& Dubinsky WP (2008) Breast cancer related proteins are present in saliva and are modulated secondary to ductal carcinoma in situ of the breast. Cancer Invest 26, 159-167.

37. Duncan MW \& Hunsucker SW (2005) Proteomics as a tool for clinically relevant biomarker discovery and validation. Exp Biol Med (Maywood) 230, 808-817.

38. Domon B \& Aebersold R (2006) Mass spectrometry and protein analysis. Science 312, 212-217.

39. Anderson L \& Hunter CL (2006) Quantitative mass spectrometric multiple reaction monitoring assays for major plasma proteins. Mol Cell Proteomics 5, 573-588.

40. Nielsen UB \& Geierstanger BH (2004) Multiplexed sandwich assays in microarray format. $J$ Immunol Methods 290, 107-120.

41. Mariman EC (2006) Nutrigenomics and nutrigenetics: the 'omics' revolution in nutritional science. Biotechnol Appl Biochem 44, 119-128.

42. Cieniewski-Bernard C, Acosta A, Dubois E, Lamblin N, Beseme O, Chwastyniak M, Amouyel P, Bauters C \& Pinet F (2008) Proteomic analysis in cardiovascular diseases. Clin Exp Pharmacol Physiol 35, 362-366.

43. Linke T, Ross AC \& Harrison EH (2004) Profiling of rat plasma by surface-enhanced laser desorption/ionization time-of-flight mass spectrometry, a novel tool for biomarker discovery in nutrition research. J Chromatogr A 1043, 65-71.

44. Roelofsen H, Balgobind R \& Vonk RJ (2004) Proteomic analyzes of copper metabolism in an in vitro model of Wilson disease using surface enhanced laser desorption/ionizationtime of flight-mass spectrometry. $J$ Cell Biochem 93, $732-740$.

45. Reymond MA \& Schlegel W (2007) Proteomics in cancer. Adv Clin Chem 44, 103-142. 